\title{
Response to: Workup following retinal artery occlusion-experience from an outpatient retina clinic and the delay in workup
}

\author{
Gautam Vangipuram $^{1} \cdot$ Kevin J. Blinder $^{1} \cdot$ Sabin Dang ${ }^{1} \cdot$ Gaurav K. Shah $^{1}$ (I)
}

Received: 1 November 2021 / Revised: 1 November 2021 / Accepted: 4 November 2021 / Published online: 11 November 2021

(c) The Author(s), under exclusive licence to Springer-Verlag GmbH Germany, part of Springer Nature 2021

\section{Dear Editor,}

We read the response by Kishore et al. to our manuscript: Workup Following Retinal Artery Occlusion-Experience from an Outpatient Retina Clinic and the Delay in Workup. One of the goals of our paper was to foster discussion among providers on management strategies in light of recent guidelines [1]. Though we acknowledge their comments, we disagree on several points.

The diagnosis of an acute central retinal artery occlusion (CRAO) represents not only a sight threatening emergency but also portends significant cardiovascular and cerebrovascular risks [2]. As alluded to by Kishore et al., our study showed it may be possible to complete a thromboembolic workup on an outpatient basis. However, our conclusion was that on average, there was a statistically significant delay in obtaining this workup through an outpatient route vs. an emergency department (ED)(13.6 vs. 2.20 days $(p=0.003)$ ). This delay may have been due to several factors, among them accessibility to specialty care, lack of communication between providers, and inadequacy in conveying the urgent nature of the condition to patients. Inaccessibility to specialty outpatient providers has only been compounded during the ongoing COVID-19 pandemic, creating a further barrier to urgent care. The recognition of this delay is crucial in understanding potential morbidity and mortality risks in this cohort. In a study, Park et. al., of 1585 patients with an acute CRAO, $165(10.4 \%)$ had a stroke or acute myocardial event within 1 year of diagnosis [3]. Notably, the incident rate ratio (IRR) was highest in the first seven days (44.51; 95\% CI, 27.07-73.20). This increased risk continued into the first 30 days after the CRAO diagnosis. This large retrospective study demonstrates the timing of workup is

Gaurav K. Shah

gkshah1@gmail.com

1 The Retina Institute, 2201 S. Brentwood Blvd., St. Louis, MO 63144, USA extremely valuable in potentially preventing these devastating complications.

If a patient's carotid imaging workup is found to be causative, timing is also essential in receiving interventional treatment in addition to medical management as Kishore et al. had outlined. Guidelines from the AHA and American Society for Vascular Surgery recommend that carotid endarterectomy be performed within 14 days from the original ischemic event, further illustrating how a delay in obtaining imaging workup may push a patient out of this critical time window [4].

Kishore et al. has suggested that only patients within a 4.5 hour window of visual symptoms be sent to an ED for consideration of tissue plasminogen activator (TPA). The administration of TPA for CRAO has shown varied outcomes, with few studies reporting a visual benefit [5]. However, aside from the 2010 EAGLE study that did not reveal a visual acuity improvement in patients receiving intra-arterial TPA vs. a placebo group, no randomized prospective control trials have demonstrated improved visual outcomes using this approach. Furthermore, access to centers able to administer local intravenous or intraarterial TPA for acute CRAO through a designated protocol outside of academic centers remains limited. Therefore, we believe the benefit in referring patients to an emergency department immediately lies in secondary prevention of future ischemic events, rather than primary visual recovery.

In our original study discussion, we referenced a 2013 survey of 281 retina specialists in which $82 \%$ indicated he or she would pursue an outpatient imaging workup for an acute RAO. However, this management paradigm has since markedly changed. In a 2021 Preferences and Trends (PAT) survey by the American Society of Retina Specialists, among 990 participants, $74.1 \%$ of US and $60.7 \%$ of international retina specialists, respectively, would refer a patient to an emergency department with an associated stroke center when a patient presents with an acute symptomatic CRAO with an embolus visible at the optic nerve head. We are 
encouraged by this trend and agree with this management based on our findings and the current literature.

\section{References}

1. Flaxel CJ, Adelman RA, Bailey ST, Fawzi A, Lim JI, Vemulakonda GA, Ying GS (2020) Retinal and ophthalmic artery occlusions preferred practice pattern ${ }^{\circledR}$. Ophthalmology 127(2):259-287

2. Chang YS, Chu CC, Weng SF et al (2015) The risk of acute coronary syndrome after retinal artery occlusion: a population based cohort study. Br J Ophthalmol 99:227

3. Park SJ, Choi NK, Yang BR et al (2015) Risk and risk periods for stroke and acute myocardial infarction in patients with central retinal artery occlusion. Ophthalmology 122:2336e 2343

4. Brott TG, Halperin JL, Abbara S, American College of Cardiology Foundation/American Heart Association Task Force on Practice Guidelines; American Stroke Association; American Association of Neuroscience Nurses; American Association of Neurological Surgeons; American College of Radiology; American Society of Neuroradiology; Congress of Neurological Surgeons; Society of Atherosclerosis Imaging and Prevention; Society for Cardiovascular Angiography and Interventions; Society of Interventional
Radiology; Society of Neuro Interventional Surgery; Society for Vascular Medicine; Society for Vascular Surgery et al (2011) 2011 ASA/ACCF/AHA/AANN/ AANS/ACR/ASNR/CNS/SAIP/SCAI/ SIR/SNIS/SVM/SVS guideline on the management of patients with extracranial carotid and vertebral artery disease: executive summary: a report of the American College of Cardiology Foundation/ American Heart Association Task Force on Practice Guidelines, and the American Stroke Association, American Association of Neuroscience Nurses, American Association of Neurological Surgeons, American College of Radiology, American Society of Neuroradiology, Congress of Neurological Surgeons, Society of Atherosclerosis Imaging and Prevention, Society for Cardiovascular Angiography and Interventions, Society of Interventional Radiology, Society of Neuro Interventional Surgery, Society for Vascular Medicine, and Society for Vascular Surgery. Vasc Med 16:35e77

5. Dumitrascu OM, Newman NJ, Biousse V (2020) Thrombolysis for central retinal artery occlusion in 2020: time is vision! J Neuroophthalmol 40(3):333-345

Publisher's note Springer Nature remains neutral with regard to jurisdictional claims in published maps and institutional affiliations. 